\title{
Current status and future directions of personalized medicine
}

\author{
Alan Haruo Bryce ${ }^{1 *}$ and Robert McWilliams²
}

\begin{abstract}
A report on the Keystone Symposia on Human Genomics and Personalized Medicine convened July 17-21, 2013, in Stockholm, Sweden.
\end{abstract}

As the first Keystone symposia dedicated to this topic, the broad agenda covered the evolution of the field, status of current research endeavors, and legal and ethical considerations. Much of the research focused on national and international efforts on large scale genomic and proteomic sequencing to develop definitive reference libraries or define rare disorders. Individual investigator projects addressed specific niches of the personalized medicine spectrum with a focus on diagnostic and predictive markers. Speakers from industry and the United States Food and Drug Administration provided perspective on the impact on drug and biomarker development. Ethics and legal experts discussed current, still developing perspectives from their fields.

\section{Genomic techniques}

Sir John Bell (Oxford University, UK), chair of the UK's Human Genomics Strategy group, laid out a thesis for the emerging role of genomics in redefining the taxonomy of disease. He argued that genome-wide association studies have allowed for the robust identification of pathways that drive phenotypes, thus allowing for a more precise definition of disease. He then outlined the UK's plan to establish a nationwide database of 100,000 genomes with a focus on pathogens, cancer, and rare diseases.

Similarly, Mathias Uhlen (Royal Institute of Technology, Sweden) described the ongoing efforts of the Human Protein Atlas (http://www.proteinatlas.org). This international effort seeks to define the human

\footnotetext{
*Correspondence: bryce.alan@mayo.edu

'Division of Hematology/Oncology, Mayo Clinic, 13400 E Shea Boulevard, Scottsdale, AZ 85259, USA

Full list of author information is available at the end of the article
}

tissue-specific proteome, with all of the information publicly available. The program uses transcriptomic analysis and antibody-based protein profiling to localize proteins within tissues. Thus far, 14,000 of the 20,500 protein-coding genes have been cataloged. It is particularly striking that only around 3,000 proteins have been found to be tissue-specific, and that a third of these are specific to the testes. Thus, cell phenotype appears to be based more on differential expression levels of proteins rather than on differences in the on/off state of the proteins.

Andrew Feinberg (Johns Hopkins, USA) gave a provocative talk placing the epigenome, rather than the genome, at the center of disease development and understanding. In studying autoimmune and neuropsychiatric disease, Feinberg showed that epigenetic plasticity may be under genetic control and may play a role in normal tissue differentiation. He identified variably methylated regions that appear disrupted in cancer, potentially providing a unifying mechanistic theory for adaptability in cancers. The epigenetic hypervariability of cancers may enable a plasticity that confers a selective growth advantage over the host.

Kelly Frazer (University of California, San Diego, USA) presented her findings on the genetic and epigenetic underpinnings of chronic lymphocytic leukemia with serial characterization of 27 patients at two time points. Interestingly, there was limited subclonal evolution across the two time points and, despite heterogeneity at the DNA level, subclones appeared to converge at the methylation and RNA levels. Moreover, the relative proportions of the subclonal populations were constant. Recalling Feinberg's hypothesis, the disease appeared to evolve through changes in methylation. These findings suggest the presence of homeostasis between subclones, which extends the concept of the tumor microenvironment to the interactions between subclones - a concept that may have implications for the therapeutic approach to tumor heterogeneity.

\section{Clinical applications of genomic techniques}

The diverse applications of genomic techniques to current clinical practice was evident at this meeting. 
Geoffrey Ginsburg (Duke University, USA) presented results of host serum gene expression profiling for the early diagnosis of infection. His assay identified asymptomatic patients with acute respiratory infections with $>95 \%$ accuracy. A second study for the detection of asymptomatic influenza A/H1N1 demonstrated 92\% accuracy. The group is now exploring similar signatures for bacterial and fungal infections.

Stephen Kingsmore (Children's Mercy Hospital, USA) and William Gahl (National Institutes of Health, USA) presented their efforts in the use of deep sequencing for undiagnosed diseases. Kingsmore demonstrated how pediatric monogenetic diseases are particularly amenable to genomic investigation, and presented his experience with over 500 undiagnosed patients. His team reached a diagnosis in nearly $40 \%$ of cases, with therapeutic potential in some cases.

Gahl presented the experiences of the National Institutes of Health Undiagnosed Disease Program. This program focuses on patients who have had extensive inconclusive evaluation elsewhere. Their software algorithm uses filters and a double reference genome derived from the patients' parents that allow the typical number of missense variants per individual, around 10,000, to be reduced to around 5 to 20 for further discussion. Using this approach, a diagnosis has been made in approximately $10 \%$ of patients, with a majority being rare diseases affecting fewer than 1 in 10,000 people.

Two studies applying genomic analyses to patients with advanced cancers were presented. Thomas Hudson (Ontario Institute for Cancer Research, Canada) reported on a multi-institutional study applying a multi-gene sequencing panel to help direct patients being considered for phase I and II clinical trials. To date, actionable mutations have been identified in 16 out of 50 patients, with matching therapy delivered in six. Three partial responses and one stable disease were reported. The panel results were available within 21 days, and the group is moving forward with a larger gene panel.

Similarly, we reported our experience with a pilot study of whole genome or exome sequencing and transcriptome sequencing (RNA sequencing) in a similar patient population. We identified actionable targets in 16 out of 30 patients. Ten patients received genomically directed therapy, and in the seven patients with results to date, three have had a partial response. The successfully treated patients had variants that included a novel variant that is not incorporated into any published cancer gene panel. Moreover, the tumor demonstrated allele-specific expression with the variant comprising $75 \%$ of transcripts, but only $11 \%$ of genome alleles. The other two responders had novel gene fusions only detectable by whole genome sequencing or RNA sequencing.

\section{Regulatory considerations and impact on drug development}

Janet Woodcock (Center for Drug Evaluation and Research, US Food and Drug Administration, USA) discussed the evolving regulatory pathway for personalized medicine. She posited that the traditional drug development pathway of phase I, II, and III trials is poorly suited for personalized medicine. The example of crizotinib's accelerated approval featured prominently throughout this presentation and others as a way to highlight the potential of larger treatment effects to allow for smaller trials; the role of the companion diagnostic as a necessary consideration in developing targeted therapy studies; and the time and cost savings of focused trials in appropriately selected patients. Woodcock made clear the Food and Drug Administration's commitment to partnering with industry and investigators to fully realize the potential of personalized medicine.

Michelle Penny (Eli Lilly and Company, USA) discussed the challenges facing industry in incorporating personalized medicine and novel biomarkers into drug development. Penny argued that the drug development process needs to be rethought to be patient-centric rather than drug-centric in design. Although $100 \%$ of surveyed pharmaceutical companies invest in biomarkers to accompany new drugs, only $10 \%$ of phase III studies currently incorporate biomarkers. Reflecting on the cost savings with crizotinib, Penny discussed the ever-falling number of drugs approved per billion US dollars invested. Although genome-based patient selection can help stem this decline, cost-effective personalized medicine will likely require that all patients be routinely screened and then directed to an appropriate therapy the patient-centric approach.

Ultimately, the meeting reflected the breadth of potential for human genomics and personalized medicine, as well as its early successes. Ongoing support from national governments, academia, and industry lend optimism to the expectation that these successes will be forerunners rather than exceptions.

Competing interests

The authors declare that they have no competing interests.

\section{Author details}

'Division of Hematology/Oncology, Mayo Clinic, 13400 E Shea Boulevard, Scottsdale, AZ 85259, USA. '2Department of Medical Oncology, Mayo Clinic, $2001^{\text {st }}$ Sreet SW, Rochester, MN 55905, USA.

Published: 29 July 2013

doi:10.1186/gm466

Cite this article as: Bryce AH, McWilliams R: Current status and future directions of personalized medicine. Genome Medicine 2013, 5:62. 